\title{
ATUAÇÃO DO ENFERMEIRO NAS LESÕES POR PRESSÃO EM PACIENTES DE UNIDADE DE TERAPIA INTENSIVA
}

\section{NURSES' PERFORMANCE IN PRESSURE INJURIES IN PATIENTS IN THE INTENSIVE CARE UNIT}

\author{
Camila Souza Rodrigues ${ }^{1} *$ Cíntia de Carvalho Silva ${ }^{2} *$ Diego Augusto Lopes Oliveira ${ }^{3}$ Janaína \\ Pereira de Melo Macêdo ${ }^{4}$ Letícia Mayara da Silva ${ }^{5} *$ Mayra Eloisa da Silva ${ }^{6} *$ Milena Larissa \\ Ferreira da Silva $^{7}$ Tainara Joana Alves de Souza ${ }^{8}$
}

\begin{abstract}
RESUMO
Objetivo: Elucidar a atuação do enfermeiro no cuidado ao cliente acometido por lesões por pressão em leitos de Unidade de Terapia Intensiva. Métodos: Revisão integrativa da literatura, feita através de coletas de dados em artigos científicos nas bases de dados LILACS, BDENF e MEDLINE, em português, inglês e espanhol, entre os anos de 2015 a 2020. Realizou-se a leitura dos títulos, resumos e dos artigos completos que respondessem ao objetivo proposto e apresentaram-se as sínteses dos resultados dos quatros artigos selecionados. A estratégia de busca seguiu a estratégia PICOS conforme pressupostos do PRISMA. Resultados: As lesões por pressão representam um problema de saúde pública em virtude do grande número de pessoas com lesão por pressão, entretanto é importante que todos os envolvidos no cuidado tenham conhecimento de como tratar de maneira adequada as lesões por pressão, a fim de evitá-las. É necessário promover educação permanente adequada para aquisição de competências no que diz respeito ao planejamento de ações, iniciado pela avaliação ao planejamento de ações, como também a prevenção e o tratamento. Conclusão: Este trabalho concluiu que o enfermeiro tem papel crucial no tratamento e na aplicação de medidas de prevenção das lesões por pressão. Assim, essa revisão possibilitou uma maior compreensão das principais ações do enfermeiro na lesão por pressão, bem como dos procedimentos a serem adotados durante o atendimento aos pacientes com essas feridas, visando sempre o melhor atendimento e o melhor tratamento possível.
\end{abstract}

Palavras-chave: Enfermagem; Lesão por Pressão; Unidade de Terapia Intensiva.

\begin{abstract}
Objective: Elucidate the role of nurses in the care of clients affected by pressure injuries in Intensive Care Unit beds. Methods: Integrative literature review, carried out through data collection in scientific articles in the LILACS, BDENF, and MEDLINE databases, in Portuguese, English, and Spanish, between the years 2015 to 2020. Titles, abstracts, and complete articles that responded to the proposed objective were read and classified as summaries of the results of the four selected papers. The search strategy followed the PICOS strategy according to PRISMA assumptions. Results: Pressure injuries represent a public health problem due to a large number of people with pressure injuries, however, everyone involved in the care must be aware of how to properly treat pressure injuries, in order to prevent them. It is necessary to promote an adequate, and permanent education for the acquisition of skills about action planning, starting with the assessment of it, as well as prevention and treatment. Conclusion: This study concluded that nurses have a crucial role in the treatment and application of measures to prevent pressure injuries. Therefore, this review has enabled a greater understanding of the main actions of nurses in pressure lesions, as well as the procedures to be adopted during the attendance to the patients with these wounds, always seeking the best care and best treatment possible.
\end{abstract}

Keywords: Nursing; Pressure Injury; Intensive Care Unit.

${ }^{1}$ Centro Universitário Tabosa de Almeida (ASCES-UNITA), Caruaru, Pernambuco, Brasil. Orcid: http://orcid.org/0000-0002-8673-4050
${ }^{2}$ Centro Universitário Tabosa de Almeida (ASCES-UNITA), Caruaru, Pernambuco, Brasil. Orcid: http://orcid.org/0000-0003-0231-601X
${ }^{3}$ Centro Universitário Tabosa de Almeida (ASCES-UNITA), Caruaru, Pernambuco, Brasil. Orcid: http://orcid.org/0000-0003-1754-7275
${ }^{4}$ Centro Universitário Tabosa de Almeida (ASCES-UNITA), Caruaru, Pernambuco, Brasil. Orcid: http://orcid.org/0000-0002-6159-4856
${ }^{5}$ Centro Universitário Tabosa de Almeida (ASCES-UNITA), Caruaru, Pernambuco, Brasil. Orcid: http://orcid.org/0000-0001-6197-7278
${ }^{6}$ Centro Universitário Tabosa de Almeida (ASCES-UNITA), Caruaru, Pernambuco, Brasil. Orcid: http://orcid.org/0000-0002-1842-3808
${ }^{7}$ Centro Universitário Tabosa de Almeida (ASCES-UNITA), Caruaru, Pernambuco, Brasil. Orcid: http://orcid.org/0000-0003-4048-5893
${ }^{8}$ Centro Universitário Tabosa de Almeida (ASCES-UNITA), Caruaru, Pernambuco, Brasil. Orcid: http://orcid.org/0000-0002-9928-3332
https://doi.org/10.31011/reaid-2021-v.95-n.35-art.1170 Rev Enferm Atual In Derme v. 95, n. 35, 2021 e-021131 


\section{INTRODUÇÃO}

A lesão por pressão (LP) é um problema cada vez mais presente em pacientes que estão internados por um longo período, principalmente em Unidade de Terapia Intensiva (UTI). São conceituadas como áreas localizadas de necrose celular que atuam sobre proeminências ósseas expostas à pressão por um período suficiente de tempo para causar isquemia tecidual. No âmbito da UTI, devido às restrições impostas aos pacientes como ficar disposto num leito com acessos venosos e muitas vezes ventilação mecânica associadas às condições clínicas graves e terapias de maior complexidade, os pacientes tornam-se vulneráveis a desenvolvêla ${ }^{1,2}$.

Nota-se que a saúde pública em geral enfrenta grandes problemas no que tange a segurança do paciente há influência cultural e de recursos disponíveis. Tendo em vista essa realidade, o Brasil que faz parte da Aliança Mundial para a Segurança do Paciente, criada pela Organização Mundial de Saúde (OMS) no ano de 2004, instituiu pelo Ministério da Saúde o Programa Nacional de Segurança do Paciente (PNSP), através da Portaria $\mathrm{n}^{\circ}$ 529, de $1^{\circ}$ de abril de 2013, com o objetivo de qualificação do cuidado em saúde em todos os estabelecimentos de saúde do território nacional $^{3,4}$.

Elucida-se que um dos desafios para o serviço de saúde são as lesões por pressão, pois quando ocorrem são consideradas um indicador de qualidade da assistência de enfermagem que está sendo prestada aos pacientes. $\mathrm{O}$ seu surgimento pode trazer implicações negativas para o paciente como dor, desconforto e infecções, que podem ocasionar ao agravamento do seu estado de saúde e até mesmo levar o paciente a óbito, assim também como poderá trazer implicações para a instituição que está ofertando a internação, pois, o processo de recuperação torna-se lento, ocorre o aumento nas taxas de infecções, consequentemente haverá o aumento de gastos para o tratamento, o dimensionamento de enfermagem torna-se mais rigoroso visto ser necessário maior disponibilidade de tempo para prestação de cuidados 5 .

Verificou-se que em situações de negligência realizadas na assistência à saúde aos pacientes com lesão por pressão, os danos causados podem resultar em óbito ou sequelas permanentes, elevando o custo e o tempo da permanência hospitalar. Todavia, quando os cuidados prestados são adequados, mantêm-se o enfoque na saúde e recuperação do paciente, ocorrendo a redução na incidência da doença e o encurtamento do tempo de tratamento e/ou hospitalização ${ }^{3}$.

Acredita-se que em relação aos cuidados realizados pela equipe de enfermagem ao paciente de UTI com risco de LP é necessário que haja uma frequência de mudanças de decúbito com o objetivo de reduzir a pressão sobre a superfície corporal e 
manter a circulação sanguínea. Considerando essa mesma perspectiva fisiológica, é importante a proteção das proeminências ósseas. Outra prática imprescindível é o cuidado nutricional, os pacientes desnutridos estão mais suscetíveis e com probabilidade duas vezes maior de desenvolverem lesões de pele as LP. O cuidado com a aplicação de emolientes na pele, pois eles substituem a função de barreira decorrente da perda da lubrificação natural ${ }^{3}$.

Os registros de ocorrência de Lesão por Pressão em pacientes críticos no Brasil variam entre $11 \%$ e $88 \%^{1}$, incidência acima do publicado em literatura internacional, que é de $4,9 \%$ a $25,1 \%$. Segundo o relatório nacional de incidentes este alto número está relacionado à assistência à saúde, entre janeiro de 2014 e julho de 2017, foram notificados 134.501 incidentes; destes, $17,6 \%$ são referentes à ocorrência de LP, sendo o estágio III prevalente nas notificações dos Núcleos de Segurança do Paciente (NSP) dos serviços de saúde do país ${ }^{6}$.

Dessa forma, a pesquisa tem por objetivo elucidar a atuação do enfermeiro no cuidado ao paciente acometido por lesões por pressão em leitos de Unidade de Terapia Intensiva (UTI).

\section{MÉTODOS}

Trata-se de um estudo bibliográfico do tipo revisão integrativa de literatura que, para sua realização, seguiram-se as seguintes etapas: Identificação do tema e seleção da questão de pergunta; Estabelecimento de critérios para inclusão e exclusão de estudos na literatura; Definição das informações a serem extraídas dos estudos selecionados; Avaliação dos estudos incluídos e apresentação da revisão/síntese do conhecimento ${ }^{5}$.

Considerou-se a seguinte questão norteadora com base nesse princípio: Qual é a atuação do enfermeiro no cuidado ao paciente com lesões por pressão em leitos de Unidade de Terapia Intensiva (UTI)? Dando seguimento foram selecionados artigos no período de 2015 a 2020, nas bases de dados a Literatura Latino-Americana/LILACS, Bases de Dados em Enfermagem/BDENF e Medical Literature Analysis and Retrieval System online/MEDLINE nos idiomas: português, inglês e espanhol. Adotaram-se os seguintes critérios de inclusão: artigos publicados em português, inglês e espanhol com os textos completos disponíveis nas bases de dados selecionadas. Constituíram-se como critérios de exclusão: artigos em que não foi possível identificar relação com a temática, editoriais, artigos de revisão e textos que exigiam pagamento para ter acesso. Utilizaram-se os seguintes descritores cruzados com o marcador booleano "AND": Enfermagem, lesão por pressão, Unidade de terapia Intensiva; Nursing, Pressure ulcer, Intensive Care Unit e Enfermería, Úlcera por presión, Unidades de Cuidados intensivos. 
Formulou-se um fluxograma do processo de seleção dos artigos, com o intuito de facilitar o entendimento e ajudar na construção deste estudo. As estratégias utilizadas para busca dos artigos foram: Inclusão dos descritores; Identificação de bases de dados e artigos com temas relacionados; Triagem (títulos repetidos, temas não relacionados, excluídos após a leitura); Elegibilidade e critérios de inclusão e exclusão, a partir das recomendações do checklist do Statement for Reporting Systematic Review and Meta-Analyses of Studies - PRISMA ${ }^{7}$.

Para organização e análise dos estudos selecionados se utilizou um instrumento, elaborado pelos pesquisadores, Em seguida, foi realizado a leitura na íntegra dos artigos para uma análise descritiva e criteriosa, estruturaram-se as informações dos estudos selecionados em um instrumento criado que contemplou: autores, título, objetivo, ano e periódico. Enquanto, o outro instrumento utilizado contemplou: título, metodologia da pesquisa, nível de evidência e síntese dos resultados.

Para a categorização do nível de evidência se considerou o tipo de estudo, utilizou-se a classificação hierárquica em sete níveis: nível I: Evidência de uma revisão sistemática ou metanálise de todos os Ensaios Clínicos Randomizados (ECR) relevantes; nível II: Evidências obtidas de ECRs bem planejados; nível III: Evidências resultantes de ensaios controlados bem delineados sem randomização; nível IV: Evidências de casos bem planejados e estudos de coorte; nível V: Evidências de revisões sistemáticas de estudos descritivos e qualitativos; nível VI: Evidências de estudos descritivos ou qualitativos únicos; nível VII: Evidências da opinião de autoridades e/ou relatos de comitês de especialistas ${ }^{8}$.

Por se tratar de uma revisão integrativa da literatura, esse estudo não necessitou da aprovação do Comitê de Ética em Pesquisa, contudo, foram considerados aspectos éticos como a citação dos autores dos artigos selecionados.

\section{RESULTADOS}

Ressalta-se que, com base no cruzamento dos descritores nas bases selecionadas, a pesquisa inicial resultou em 113 publicações. Restaram-se após classificados os critérios de inclusão, 12 artigos nos idiomas em português e inglês. Excluíram-se 8 por não atenderem os critérios de elegibilidade previamente estabelecidos, restando, assim, 4 artigos analisados pela leitura em texto completo e incluídos nesta revisão integrativa.

Acrescenta-se que $(n=1)$ artigos foram encontrados na base de dados do BDENF, $(n=2)$ no LILACS e $(n=1)$ no MEDLINE. Predomina-se entre as publicações o idioma português (50\%), inglês $(50 \%)$ e espanhol $(0 \%)$. 
Figura 1: Fluxograma utilizado para seleção dos estudos e identificação, elaborado a partir da recomendação PRISMA. Caruaru/PE, 2021.

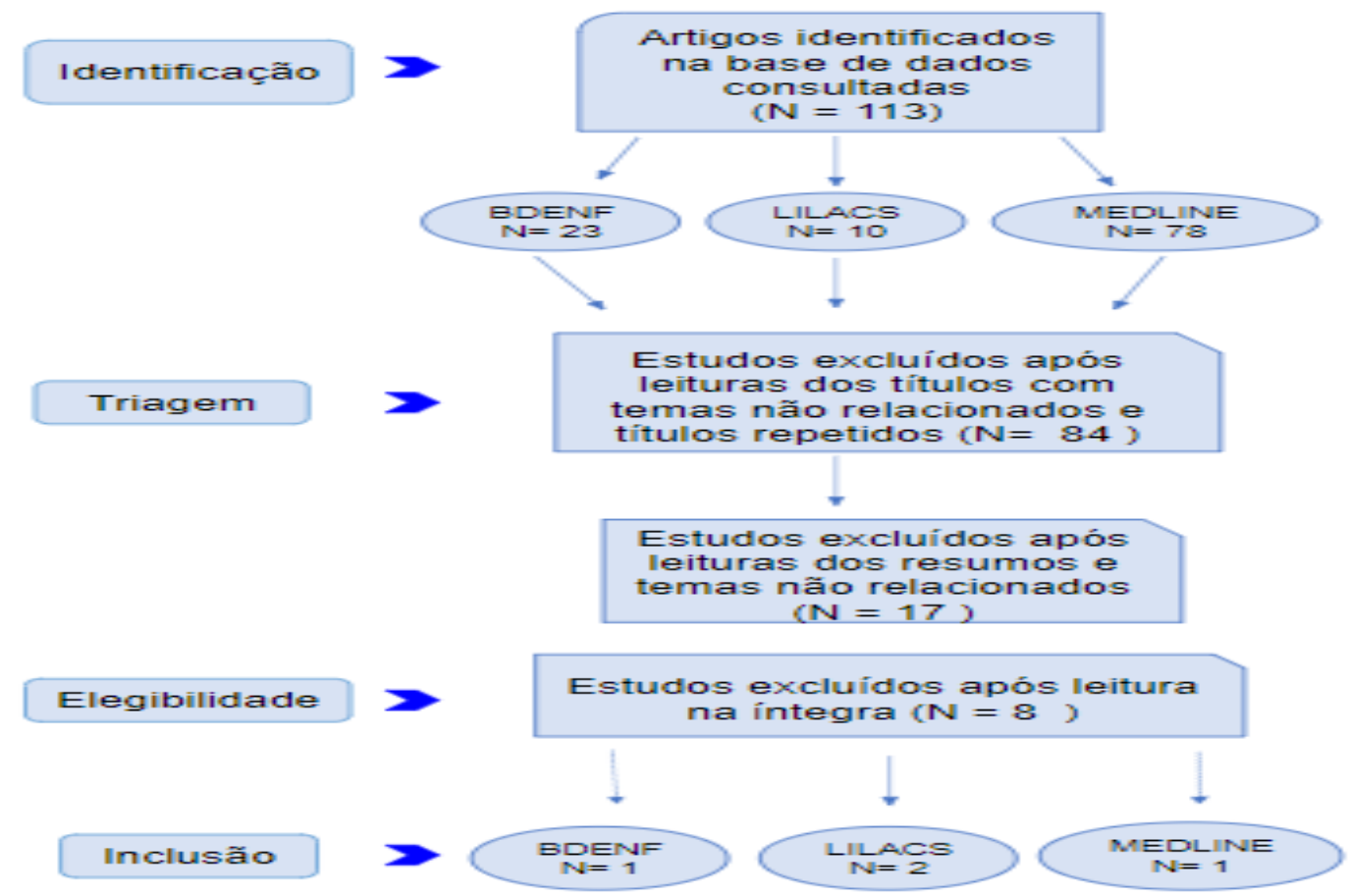

Fonte: Os Autores

Quadro 1: Caracterização dos estudos, após critérios de elegibilidade e conforme pergunta condutora. Caruaru, 2021.

\begin{tabular}{|c|c|c|c|c|c|}
\hline Id & Autores & Título & Objetivo & Ano & Periódico \\
\hline 01 & $\begin{array}{l}\text { Baron, Miriam } \\
\text { Viviane; Reuter, } \\
\text { Cézane Priscila; } \\
\underline{\text { Burgos, Miria }} \\
\underline{\text { Suzana; Cavalli, }} \\
\text { Veniria; } \\
\text { Brandenburg, } \\
\text { Cristine; Krug, } \\
\underline{\text { Suzane Beatriz }} \\
\text { Frantz. }\end{array}$ & $\begin{array}{l}\text { Experimental } \\
\text { study with } \\
\text { nursing staff } \\
\text { related to the } \\
\text { knowledge about } \\
\text { pressure ulcers. }\end{array}$ & $\begin{array}{l}\text { To compare the scores of } \\
\text { knowledge in teams } \\
\text { participating or not } \\
\text { participating in educational } \\
\text { interventions about pressure } \\
\text { ulcers. }\end{array}$ & 2016 & $\begin{array}{l}\frac{\text { Rev Lat Am }}{\text { Enfermagem; }} \\
\text { 24: e 2831, } \\
20161121 .\end{array}$ \\
\hline 02 & Vasconcelos, & Ações de & Avaliar as ações dos & 2017 & $\underline{\text { Esc. Anna }}$ \\
\hline
\end{tabular}




\begin{tabular}{|c|c|c|c|c|c|}
\hline & $\begin{array}{l}\text { Josilene de Melo } \\
\underline{\text { Buriti; }} \text { Caliri, Maria } \\
\underline{\text { Helena Larcher. }}\end{array}$ & $\begin{array}{l}\text { enfermagem } \\
\text { antes e após um } \\
\text { protocolo de } \\
\text { prevenção de } \\
\text { lesões por } \\
\text { pressão em } \\
\text { terapia intensiva. }\end{array}$ & $\begin{array}{l}\text { profissionais de } \\
\text { enfermagem, antes e após } \\
\text { utilização de protocolo de } \\
\text { prevenção de lesões por } \\
\text { pressão, em Unidade de } \\
\text { Terapia Intensiva. }\end{array}$ & & $\begin{array}{l}\text { Nery Rev. } \\
\text { Enferm; 21(1): } \\
\text { e20170001, } \\
\text { 2017. tab }\end{array}$ \\
\hline 03 & $\begin{array}{l}\text { Soares, Rhea Sílvia } \\
\underline{\text { Avila; }} \underline{\text { Lima, }} \\
\underline{\text { Suzinara Beatriz }} \\
\underline{\text { Soares de; }} \\
\underline{\text { Camponogara, }} \\
\underline{\text { Silviamar; Eberhard, }} \\
\underline{\text { Thaís Dresch; }} \\
\underline{\text { Fonseca, Graziele }} \\
\text { Gorete Portella da; } \\
\underline{\text { Kessler, Marciane. }}\end{array}$ & $\begin{array}{l}\text { Significado do } \\
\text { protocolo de } \\
\text { úlcera por } \\
\text { pressão: } \\
\text { qualificando a } \\
\text { gerência do } \\
\text { cuidado do } \\
\text { enfermeiro. }\end{array}$ & $\begin{array}{l}\text { Compreender o significado } \\
\text { do protocolo assistencial de } \\
\text { Úlceras por Pressão para } \\
\text { enfermeiros no } \\
\text { gerenciamento do cuidado } \\
\text { de enfermagem. }\end{array}$ & 2017 & $\begin{array}{l}\text { Enferm. foco } \\
\underline{\text { Brasília); }} \\
\text { 8(3): 19-24, } \\
\text { nov.-2017. } \\
\text { graf }\end{array}$ \\
\hline 04 & $\begin{array}{l}\text { Nahla Tayyiba } \\
\text { Fiona Boyer, Peter } \\
\underline{\text { A.Lewis }}\end{array}$ & $\begin{array}{l}\text { Implementing a } \\
\text { pressure ulcer } \\
\text { prevention } \\
\text { bundle in an } \\
\text { adult intensive } \\
\text { care }\end{array}$ & $\begin{array}{l}\text { Os objetivos deste estudo } \\
\text { foram avaliar a } \\
\text { implementação de uma } \\
\text { série de componentes do } \\
\text { pacote de cuidados de } \\
\text { intervenção de alto impacto, } \\
\text { direcionados à prevenção } \\
\text { do desenvolvimento de } \\
\text { UPs, na UTI, e avaliar a } \\
\text { eficácia das estratégias } \\
\text { utilizadas para melhorar a } \\
\text { conformidade da } \\
\text { implementação. }\end{array}$ & 2016 & $\begin{array}{l}\text { SciencDirect } \\
(2016)\end{array}$ \\
\hline
\end{tabular}

Fonte: Os autores 
Quadro 2: Descrição dos artigos selecionados, após leitura completa e conforme pergunta condutora. Caruaru/PE, 2021.

\begin{tabular}{|c|c|c|c|c|}
\hline Id & Título & Metodologia & $\begin{array}{r}\text { Nível de } \\
\text { evidência }\end{array}$ & Síntese do resultado \\
\hline 01 & $\begin{array}{l}\text { Significado do } \\
\text { protocolo de } \\
\text { úlceras por } \\
\text { pressão: } \\
\text { qualificando a } \\
\text { gerência do } \\
\text { cuidado do } \\
\text { enfermeiro. }\end{array}$ & $\begin{array}{l}\text { Utilizou-se a Teoria da } \\
\text { Complexidade como } \\
\text { referencial teórico e a } \\
\text { estudo qualitativo nos } \\
\text { Dados como referencial } \\
\text { metodológico. }\end{array}$ & Nível IV & $\begin{array}{l}\text { Na perspectiva do cuidado } \\
\text { complexo, os enfermeiros se } \\
\text { apoiam no protocolo como } \\
\text { instrumento que padroniza os } \\
\text { cuidados, mantendo um cuidado } \\
\text { flexível e humano, mais } \\
\text { subjetivo que objetivo no } \\
\text { momento da sua execução, } \\
\text { considerando as expectativas } \\
\text { dos pacientes. A partir da } \\
\text { interconexão das categorias, o } \\
\text { fenômeno central desvelado que } \\
\text { representa a Matriz Teórica foi } \\
\text { significando o protocolo de } \\
\text { Úlcera por Pressão como } \\
\text { instrumento de qualificação } \\
\text { para o cuidado gerencial do } \\
\text { enfermeiro. }\end{array}$ \\
\hline 02 & $\begin{array}{l}\text { Experimental } \\
\text { study with } \\
\text { nursing staff } \\
\text { related to the } \\
\text { knowledge about } \\
\text { pressure ulcers }\end{array}$ & $\begin{array}{l}\text { Um estudo quantitativo } \\
\text { com delineamento } \\
\text { experimental. }\end{array}$ & Nível III & $\begin{array}{l}\text { As estratégias utilizadas para } \\
\text { operacionalizar a intervenção } \\
\text { educativa realizadas neste } \\
\text { estudo são importantes meios } \\
\text { para a difusão das } \\
\text { recomendações para a } \\
\text { prevenção da UP. Podem ser } \\
\text { utilizadas por gerentes de } \\
\text { Enfermagem, gestores políticos, } \\
\text { educadores e pesquisadores }\end{array}$ \\
\hline
\end{tabular}




\begin{tabular}{|c|c|c|c|c|}
\hline & & & & $\begin{array}{l}\text { como estratégias facilitadoras } \\
\text { para o desenvolvimento e a } \\
\text { implementação de programas } \\
\text { educacionais para a prevenção } \\
\text { da UP. Dos grupos que } \\
\text { participaram, o resultado foi de } \\
74,1 \% \text { no grupo de intervenção } \\
\text { e } 76,0 \% \text { no grupo de controle e, } \\
\text { no momento pós-intervenção, } \\
\text { foi de } 87,0 \% \text { no grupo que } \\
\text { sofreu intervenção educativa, } \\
\text { considerando que o grupo de } \\
\text { controle foi de } 79,1 \% \text { O grupo } \\
\text { que apresentou habilidades } \\
\text { educativas não atingiu uma } \\
\text { média adequada de } 90 \% \text { dos } \\
\text { valores para teste. }\end{array}$ \\
\hline $\mathbf{0 3}$ & $\begin{array}{l}\text { Implementing a } \\
\text { pressure ulcer } \\
\text { prevention bundle } \\
\text { in an adult } \\
\text { intensive care }\end{array}$ & $\begin{array}{l}\text { Um estudo observacional } \\
\text { prospectivo foi utilizado. } \\
\text { As estratégias de } \\
\text { implementação incluíram } \\
\text { educação regular, } \\
\text { treinamento, auditoria e } \\
\text { feedback e a presença de } \\
\text { um campeão na UTI. }\end{array}$ & IV & $\begin{array}{l}\text { As estratégias de } \\
\text { implementação utilizadas } \\
\text { mostraram um impacto positivo } \\
\text { na conformidade. Avaliar a } \\
\text { conformidade da } \\
\text { implementação é fundamental } \\
\text { para alcançar o resultado } \\
\text { desejado. Os participantes do } \\
\text { estudo demonstraram um alto } \\
\text { nível de adesão à } \\
\text { implementação do pacote de } \\
\text { prevenção de UP ( } 78,1 \%) \text {, com } \\
100 \% \text { de aceitação pelos } \\
\text { participantes. }\end{array}$ \\
\hline 04 & Ações de & Estudo observacional, & IV & Os resultados obtidos neste \\
\hline
\end{tabular}




\begin{tabular}{|l|l|l|l|l|}
\hline enfermagem antes & prospectivo, comparativo, \\
e após um & do tipo antes e depois, \\
protocolo de & com abordagem & & estudo mostraram que a \\
prevenção de & quantitativa, realizado em \\
lesões por pressão & hospital de ensino, na \\
em terapia & $\begin{array}{l}\text { Paraíba. Foram } \\
\text { interara construção e }\end{array}$ \\
intensiva. & $\begin{array}{l}\text { implementação do protocolo de } \\
\text { prevenção de LP, com parceria } \\
\text { enfermagem durante 38 } \\
\text { banhos no leito antes e 44 } \\
\text { depois do protocolo. }\end{array}$ & $\begin{array}{l}\text { influenciou a adesão ao uso das } \\
\text { recomendações baseadas em }\end{array}$ \\
& & $\begin{array}{l}\text { evidências científicas, pelos } \\
\text { profissionais de enfermagem na } \\
\text { UTI estudada. }\end{array}$ \\
\hline
\end{tabular}

Fonte: Os autores

\section{DISCUSSÃO}

Ressalta-se que a permanência do paciente na Unidade de Terapia Intensiva (UTI), o torna vulnerável a vários procedimentos que são desenvolvidos com o objetivo de estabilizar algum quadro clínico e durante este tempo de permanência podem surgir as Lesões por Pressão. A sua ocorrência está geralmente associada a um conjunto de fatores extrínsecos tais como: pressão, fricção, cisalhamento e umidade; e intrínsecos como: déficit nutricional, presença de edema, idade, imobilidade e patologia de base $^{9}$.

Identifica-se que após a análise e da leitura dos artigos, evidenciou-se a importância da discussão desta temática acerca da assistência, especialmente a elaboração e execução de medidas de prevenção da lesão por pressão. A utilização da Escala de Braden pode se tornar um grande aliado do enfermeiro para aumentar a qualidade do serviço proporcionado à pessoa com lesão por pressão, pois permite conhecer o seu perfil e direciona a sistematização do cuidado. Para que a utilização da escala se torne efetiva, o profissional deve estar devidamente capacitado, assegurando para que não haja situações limitantes a partir das interpretações e pontuações dos escores dos avaliadores. Para uma avaliação adequada, além de uma abordagem estruturada, como no caso da Braden, é essencial o juízo clínico, onde uma não substitui a outra ${ }^{10}$. Nesse sentido, a aplicação da Escala de Braden pelo profissional enfermeiro direciona as intervenções de enfermagem na prevenção de Lesão por Pressão.

Sabe-se que no papel do enfermeiro, é necessária uma formação adequada para a realização de competências quanto ao planejamento de ações, iniciado pela avaliação, prevenção e tratamento, além da educação em saúde dos pacientes e seus 
familiares, a fim de melhorar a assistência prestada, bem como a qualidade de vida ${ }^{11}$.

O desenvolvimento deste tipo de lesão leva a inúmeras consequências negativas que refletem no sofrimento físico e emocional para o paciente, além de elevar o risco de complicações e comorbidades a curto, médio e longo prazo. Para o sistema de saúde, resulta em maiores custos diante da necessidade da permanência prolongada no serviço, bem como maior investimento de recursos materiais e humanos para assistência ao paciente ${ }^{12}$.

Observou-se que o enfermeiro necessita de uma educação adequada para aquisição de competências no que diz respeito a planejamento de ações, a prevenção e o tratamento, a fim de melhorar a assistência prestada, bem como a qualidade de vida dos pacientes. Neste sentido, a utilização de protocolos na gerência do cuidado de enfermagem ao paciente com LP são ferramentas importantes e de impacto no controle da incidência, reduzindo complicações, tempo de internação e redução da mortalidade associada às LP, no entanto, apresentam elevados custos para sua elaboração, implementação, avaliação e manutenção ${ }^{13}$.

Entende-se que os protocolos assumem posição de facilitador do cuidado, são rotinas e ações de cuidado e gestão dos serviços ou equipes de saúde. São instrumentos elaborados a partir da prática baseada em evidências que permitem um cuidado de acordo com as melhores opções e têm impacto em práticas de melhor qualidade e segurança para o paciente. Dessa forma, os enfermeiros devem estar atentos para identificar e enfrentar situações que exigem novas estratégias que contribuam para a qualidade do cuidado. É considerado um esquema terapêutico que orienta a equipe quanto à avaliação e aplicação do tratamento mais adequado para a reabilitação integral do paciente. A avaliação deve levar em conta a causa, tempo de existência, presença ou ausência de infecção na lesão, e deve seguir uma ordem lógica de classificação, escolha do curativo adequado, acompanhamento e reavaliação da lesão, bem como a utilização de padrões de classificações de riscos que auxiliam na prevenção da ocorrência dessas lesões, que não podem ser tratadas apenas pelo que é possível ver, comorbidades e alterações fisiológicas devem ser corrigidas em conjunto com o tratamento local ${ }^{14,15}$.

Destaca-se que os enfermeiros têm o papel fundamental na avaliação e no tratamento dessas lesões e devem sensibilizar, incentivar e treinar a equipe para que sigam padrões definidos de tratamento, tem também a responsabilidade de prever e prover recursos humanos, materiais e estruturais, e de implantar medidas preventivas para que assim tenhamos melhores resultados ${ }^{16}$.

As limitações apresentadas foram a quantia de fontes atuais na literatura em 
relação à temática, indicando a produção de estudos com metodologias de evidência mais elevada na área, que corroborem para resultados mais específicos no cuidado de enfermagem, desde a prevenção e promoção de saúde à continuidade de uma boa qualidade de vida durante o tratamento as LP.

\section{CONCLUSÃO}

Conclui-se com este trabalho que o enfermeiro tem papel crucial tanto no tratamento das lesões por pressão, quanto para a escolha do tratamento mais adequado, e na aplicação de medidas de prevenção dessas lesões. É preciso que o enfermeiro tenha conhecimento sobre o processo que envolve o tratamento do paciente e que desenvolva um protocolo de atendimento para pacientes com lesões por pressão para que toda a equipe envolvida possa seguir os mesmos padrões de avaliação e tratamento.

Evidenciou-se que a análise dos estudos proporcionou reflexão no tocante à importância das ações de enfermagem na assistência ao cliente em tratamento das lesões por pressão, por demonstrar que sua realização viabiliza impactos negativos, desde a manutenção da saúde à prevenção de complicações mais severas. Os estudos identificados foram em sua maioria de publicações internacionais, e ainda que os artigos incluídos sejam nos idiomas português e inglês, é perceptível a deficiência do Brasil no investimento sobre o conhecimento de como o enfermeiro atua nesse cenário, bem como a necessidade de níveis de evidência mais altos para haver uma maior confiança de que as publicações são o mais próximo possível da realidade.

Salienta-se que as limitações apresentadas foram a quantia de fontes atuais na literatura em relação à temática, indicando a produção de estudos com metodologias de evidência mais elevada na área, que corroborem para resultados mais específicos no cuidado de enfermagem, desde a prevenção e promoção de saúde à continuidade de uma boa qualidade de vida durante o tratamento as LP.

\section{REFERÊNCIAS}

1. Gomes FSL, et al. Avaliação de risco para úlcera por pressão em pacientes críticos. Rev. Esc. Enferm. USP 2011; 45(2): 313-318.

2. Barbosa TP, Beccaria LM, Poletti NAA. Avaliação do risco de úlcera por pressão em UTI e assistência preventiva de enfermagem. Rev Enferm UERJ [Internet]. 2014 maio-jun [acesso em 2017 nov 18];22(3):353- 8. Disponível em: http://www.facenf.uerj.br/v22n3/v22n3a10.pd f. Acesso em: 14 de maio de 2020.

3. Busanello et tal. Cuidados de enfermagem ao paciente adulto: prevenção de lesões cutaneomucosas e segurança do paciente. Disponível em: https://periodicos.ufsm.br/index.php/reufsm/a rticle/view/16310/pdf. Acesso em: 14 de maio de 2020.

4. Brasil, Ministério da Saúde. Portaria $n^{\circ}$ 529, DE $1^{\circ}$ de abril de 2013. Disponível em:https://bvsms.saude.gov.br/bvs/saudelegis/ gm/2013/prt0529_01_04_2013.html. Acesso em: 14 de maio de 2020.

5. Manganelli et tal. Intervenções de enfermeiros na prevenção de lesão por 
pressão em uma unidade de terapia intensiva. Disponível

em:https://periodicos.ufsm.br/reufsm/article/v iew/33881/pdf\#. Acesso em. 14 de maio de 2020.

6. Agência Nacional de Vigilância sanitária (ANVISA). Práticas seguras para prevenção de Lesão por Pressão em serviços de saúde. Nota técnica GVIMS/GGTES n. 03/2017. [Internet] 2017. Available from: https://www20.anvisa.gov.br/segurancadopaci ente/index.php/alertas/item/nota-técnicagvims-ggtes-03-2017.

7. Galvão TF, Pansani, TSA, Harrad D. Principais itens para relatar revisões sistemáticas e meta-análises: a recomendação PRISMA. Epidemiol Serv Saude [Internet]. 2015 [cited 2018 Mai 22]; 24(2):335-42. Available from: http://www.scielo.br/pdf/ress/v24n2/22379622- ress-24-02-00335.pdf.

8. Melnyk BM, Gallagher-Ford L, FineoutOverrholt E. Implementing the evidencebased practice (EBP) competencies in healthcare: a practical guide to improving quality, safety, and outcomes. In: Sigma Theta Tau International. Indianapolis: 2016. p.78-9.

9. Alencar GSA, Silva NM, Assis EV, Sousa MNA, Pereira JLF, Oliveira WB, et al. Lesão por pressão na unidade de terapia intensiva: incidência e fatores de riscos. Rev Nursing [Internet]. 2018 [cited 2018 Jun 06]; 21(239):2124-8. Available from: http://www.revistanursing.com.br/revistas/23 9-

Abril2018/lesao_por_pressao_na_unidade_de terapia_intensiva.pdf.

10. Silva ALM, Rached CDA, Liberal MMC. A utilização da escala de braden como instrumento preditivo para a prevenção de lesão por pressão. Rev. Direito em Foco, 2019.

11. Carneiro CM, Sousa FB, Gama FN. Tratamento de feridas: assistência de enfermagem nas unidades de atenção primária à saúde. Rev Enferm Integrada. 2010; 3(2):494-505.
12. Moraes JT, Borges EL, Lisboa CR, Cordeiro DCO, Rosa EG, Rocha NA. Conceito e classificação de lesão por pressão: atualização do National Pressure Ulcer Advisory Panel. Enferm Cent O Min. 2016 [cited 2018 Feb 12]; 6(2):2292-306. Available from: http://dx.doi.org/10.19175/recom.v6i2.1423.

13. Lima AFC, Castilho V, Rogenski NMB, Baptista CMC, Rogenski KE. Implementation costs of a prevention protocol for pressure ulcers in a university hospital. Rev. Eletr. Enf. [Internet]. 2015 out./dez.;17(4). Disponível em:

http://dx.doi.org/10.5216/ree.v17i4.31051.

14. Camelo, SHH, Soares, MI, Chaves, LDP, Rocha, FLR, Silva, VLS. Nurse managers at a teaching hospital: training, responsibilities and challenges. Revista Enfermagem UERJ. 2016 24(3), e11637.

15. Lima AFC, Castilho V. Body Mobilization for prevention of pressure ulcers: direct labor costs.Rev Bras Enferm. 2015;68(5):647-52.

16. Tiago F, Oliveira CT, Silva SS, Melo AS. Conhecimento dos graduandos de enfermagem reativo à úlcera por pressão: um estudo no cenário privado. Nursing. 2012 ; 14(164):21-8.

\section{Autor correspondente}

Mayra Eloisa da Silva Endereço: Avenida João Bartolomeu Torres, Cidade Jardim, Caruaru/PE, Nº745, CEP 55021- 280 Celular: (+55) 81.99877-3058 email: mayra_eloisa@hotmail.com

Submissão: 2021-07-06

Aprovado: 2021-08-28 